\title{
Le phénomène de résistance multiple aux anti-cancéreux : les gènes MDR et la P-gp
}

La résistance aux cytostatiques est l'obstacle majeur au traitement de nombreux cancers. Les causes d'échecs des chimiothérapies sont multiples, la principale étant une résistance intrinsèque de la cellule tumorale. La reconnaissance d'une glycoprotéine de $170 \mathrm{kDa}$ (la P-gp) à la surface de cellules de lignées tumorales rendues résistantes à des cytostatiques de mode d'action différent, a permis de décrire un phénotype de cellules "multirésistantes" (MDR, pour multidrug resistance). Cette $\mathrm{P}$ $\mathrm{gp}$, codée par le gène $m d r 1$ chez l'homme, a un rôle de pompe, chassant hors de la cellule les cytostatiques. Sa responsabilité dans la résistance clinique des tumeurs humair ss est suspectée, en raison de son expression souvent élevée dans des cancers d'emblée ou devenus chimiorésistants. Des essais thérapeutiques d'inhibition de cette $P$-gp sont actuellement en cours.

\section{Jean-Pierre Marie}

\section{ADRESSE}

J.-P. Marie : praticien hospitalier, professeur à la faculté de médecine Paris VI. Service d'hématologie, Hôtel-Dieu de Paris, 1, place du Parvis-Notre-Dame, 75181 Paris Cedex 04, France.

$\mathrm{m} / \mathrm{s} n^{\circ} 5$ vol. 6 , mai 90 a résistance multiple aux anti-cancéreux (MDR) est définie comme étant une résistance croisée, étendue à des cytostatiques de famille et de mode d'action divers.

Le phénomène "MDR " a été mis en évidence par l'observation des lignées tumorales animales exposées de façon continue et prolongée à des doses croissantes d'un cytostatique [revues: 1,2]. La constatation que ces lignées étaient devenues d'emblée résistantes à d'autres cytostatiques, auxquelles elles étaient exposées pour la première fois, a conduit Biedler et Riehm, dès 1970, à envisager un mécanisme commun de résistance.

Les médicaments intéressés par le phénomène MDR contiennent généralement un noyau aromatique hydrophobique et sont issus de plantes ou de micro-organismes. Ainsi, des produits aussi importants que des anthracyclines (doxorubicine et daunorubicine), les poisons du fuseau (vincristine, vinblastine), les épipodophyllotoxines (étoposide, ténoposide), l'actinomycine $\mathrm{D}$, sont intéressés par ce phénomène de résistance croisée (Tableau I, page suivante).

La pharmacocinétique des cytostatiques a montré que ceux-ci entraient normalement dans les cellules MDR, mais que leur rétention intracellulaire était significativement diminuée par rapport à celle de la lignée parentale sensible. Cet efflux est actif, dépendant de la présence d'ATP.

Le phénomène MDR est associé dans ces modèles à l'expression d'une glycoprotéine de membrane appelée 


\begin{tabular}{|c|c|}
\hline \multicolumn{2}{|c|}{$\begin{array}{c}\text { Tableau I } \\
\text { CYTOSTATIQUES ET P-gp }\end{array}$} \\
\hline Extraction assurée par la P-gp & Non influencée par la P-gp \\
\hline $\begin{array}{l}\text { Anthracyclines } \\
\text { daunorubicine } \\
\text { adriamycine }\end{array}$ & $\begin{array}{l}\text { Alkylants } \\
\text { cyclophosphamide } \\
\text { chloraminophène }\end{array}$ \\
\hline $\begin{array}{l}\text { THP-adriamycine } \\
\text { Alcaloïdes de pervenche }\end{array}$ & $\begin{array}{l}\text { Moutardes à l'azote et dérivés } \\
\text { nitrosourées }\end{array}$ \\
\hline $\begin{array}{l}\text { vincristine } \\
\text { vinblastine }\end{array}$ & $\begin{array}{l}\text { Analogues des bases puriques et } \\
\text { pyrimidiques }\end{array}$ \\
\hline $\begin{array}{l}\text { Mitoxantrone } \\
\text { Amsacrine }\end{array}$ & $\begin{array}{l}\text { 6-mercaptopurine } \\
\text { 6-thioguanine }\end{array}$ \\
\hline $\begin{array}{l}\text { Épipodophyllotoxines } \\
\text { VP16 } \\
\text { VM26 }\end{array}$ & $\begin{array}{l}\text { cytosine arabinoside } \\
\text { Bléomycine }\end{array}$ \\
\hline Actinomycine $D$ & Corticoïdes \\
\hline
\end{tabular}

4. Roninson IB, Abelson HT, Housman tion of specific DNA sequence correlates with multidrug resistance in Chinese hamster cells. Nature $1984 ; 309: 626-9$.

5. Riordan JR, Deuchars K, Kartner N, Alon N, Trent J, Ling V. Amplification of P-glycoproteine genes in multidrug-resistant mammalian cell lines. Nature $1985 ; 316$ : 817-9.

6. Gros P, Neriah YB, Croop JM, Housman DE. Isolation and expression of a complementary DNA that confers multidrug resistance. Nature 1986 ; 323 : 728-31.

7. Ueda K, Clark DP, Chen C, Roninson IB, Gottesman MM, Pastan I. The human multidrug resistance $(m d r 1)$ gene : cDNA cloning and transcription initiation. $J$ Biol Chem 1987; 262 : 505-8.

8. Gerlach JH, Endicott JA, Juranka PF et al. Homology between P-glycoprotein and a bacterial haemolysin transport protein suggests a model for multidrug resistance. Nature 1986 ; 324 : 485-9.

9. Ueda K, Cardarelli C, Gottsman MM, Pastan I. Expression of a full-length cDNA for the human $m d r l$ gene confers resistance to colchicine, doxorubicin, and vinblastine. Proc Natl Acad Sci USA 1987 ; 84 : 3004-8.

10. Galski $H$, Sullivan M, Willingham MC, et al. Expression of a human multidrug resistant cDNA (MDR1) in the bone marrow of transgenic mice : resistance to daunomycin-induced leukopenia. Mol Cell Biol 1989 ; 9 : 4357-63.

11. Kartner N, Evernden Porelle D, Bradley $G$, Ling V. Detection of P-glycoprotein in multidrug resistant cell lines by monoclonal antibodies. Nature $1985 ; 316$ : 820-3.

12. Raymond M, Gros P. Mammalian multidrug-resistance gene : correlation of exon organization with structural domains and duplication of ancestral gene. Proc Natl Acad Sci USA 1989 ; 86 : 6488-92.

P-glycoprotéine (P-gp ; $\mathrm{P}$ pour perméabilité), décrite par Ling dans une lignée de cellules ovariennes de hamster chinois rendues multirésistantes par exposition à la colchicine [3]. Cette protéine, absente des membranes des cellules parentales sensibles aux drogues, est trouvée dans de nombreuses lignées ayant un même profil de résistance. Un anticorps monoclonal dirigé contre la $\mathrm{P}$ gp (le C219) par injection de membranes plasmiques de cellules MDR à des souris a pu être produit. Grâce à cet anticorps, il a pu être montré que la structure de cette P-gp était très conservée au cours de l'évolution. Indépendamment, plusieurs équipes, travaillant sur l'ADN de lignées hautement résistantes d'origine murine puis humaine ont amplifié, grâce aux techniques de renaturation de l'ADN en gel d'agarose [4, 5], des séquences d'ADN spécifiquement retrouvées dans le génome de ces lignées. Les $\mathrm{ADNc}$ codant pour les P-gp murines et humaines ont été isolées en 1986 et sont pratiquement identiques $[6,7]$. Une grande homologie de séquence a été retrouvée entre la P-gp et l'hémolysine B protéine de transport de la paroi bactérienne, qui joue un rôle dans les processus d'excrétion [8].
La responsabilité de la $\mathrm{P}$-gp dans le phénotype de multirésistance repose sur plusieurs constatations : dans la plupart des lignées, l'expression du gène $m d r 1$ est proportionnelle au degré de résistance; une liaison directe des cytostatiques à la $\mathrm{P}$-gp a pu être démontrée par photoaffinité ; enfin et surtout la transfection de cellules sensibles, par le gène $m d r 1$, confère le phénotype de multirésistance avec expression de la P-gp [9, 10].

\section{P-glycoprotéine}

La taille des P-gp s'étend de 120 à 220 kilodaltons (kDa), mais est le plus souvent de 170 à $180 \mathrm{kDa}$. Certains anticorps monoclonaux reconnaissent les P-gp du hamster, de la souris et de l'homme, démontrant la conservation de structure entre ces espèces [11]. Dans les lignées multirésistantes à partir desquelles la $\mathrm{P}$-gp a été isolée, on trouve 100000 à 200000 molécules de P-gp par cellule ; la demi-vie de cette protéine est évaluée à 72 heures.

La P-gp humaine est composée de 1280 acides aminés, qui s'organisent en deux séquences symétriques (figure 1), évoquant la duplication d'un gène ancestral [12]. L'existence de 12 régions hydrophobes trans- 


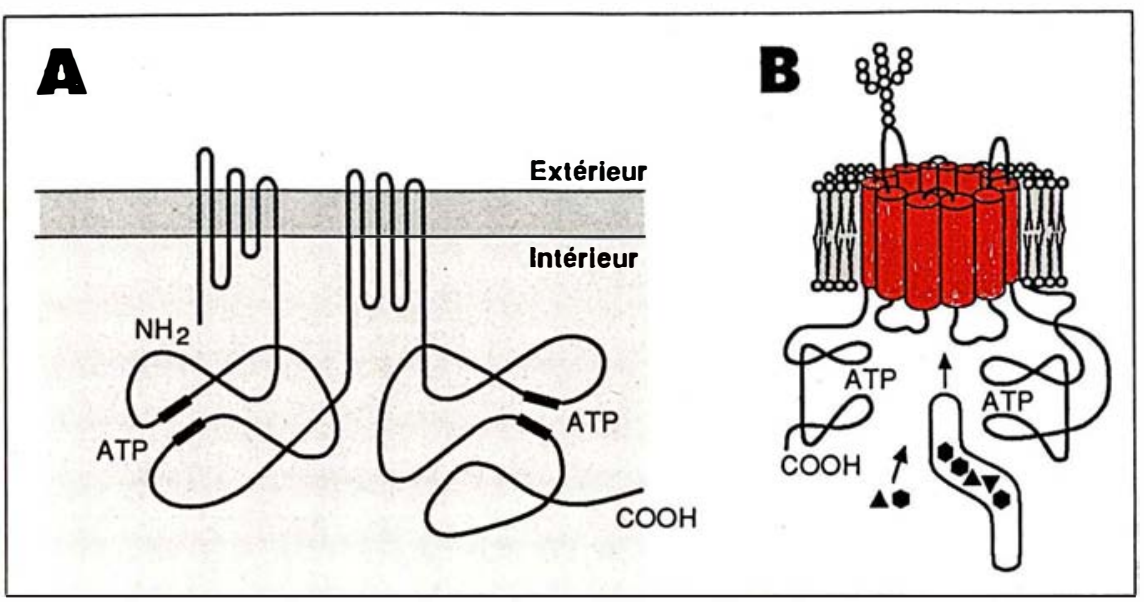

Figure 1. Schéma de la P-gp. A configuration transmembranaire et sites ATP (d'après J.A. Endicott et V. Ling). B arrangement tridimensionnel de la protéine montrant le site de glycosylation (d'après R.L. Juliano et V. Ling). sont pas connues. Le rôle fonction- membranaires lui donne une configuration caractéristique de protéine assurant un transport transmembranaire. La partie intra-cytoplasmique contient deux sites de liaison à l'ATP, suggérant que le transport des drogues vers l' extérieur est bien un phénomène actif. Une boucle située à la surface externe est glycosylée, expliquant les variations de poids moléculaire.

L'existence d'une liaison physique entre la P-gp et ses substrats a été démontrée par des expériences de photoaffinité, mais les zones de liaison avec les substrats expulsés ne nel respectif de chaque partie de la protéine est analysé par transfection d'ADNc P-gp comportant des délétions partielles [13].

Le mécanisme d'action des P-gp semble être essentiellement une diminution de l'accumulation des cytostatiques intracellulaires, par une expulsion active [13] ou par une redistribution intracellulaire [14] des médicaments intéressés. Il n'a pas été

décrit de transporteur se liant au substrat et l'apportant jusqu'à la P-gp.

\section{Gènes MDR}

La famille des gènes $M D R$ ne comporte que deux gènes chez l'homme ( $m d r 1$ et $m d r 2, m d r 1$ étant seul responsable du phénotype MDR) et trois chez les rongeurs (Tableau II). Les gènes $M D R$ se trouvent sur le chromosome 7 chez l'homme [15] et ne sont séparés que par $330 \mathrm{~kb}$. Le gène $m d r 1$ s'étend sur plus de $100 \mathrm{~kb}$, alors que l'ARNm ne mesure que $4,5 \mathrm{~kb}$ (4 $669 \mathrm{pb})$. Il y a 29 exons et 28 introns [16], dont l'un dépasse $40 \mathrm{~kb}$. Ce gène comporte deux sites majeurs d'initiation de la transcription, situés environ 140 bases en amont de la séquence ATG d'initiation de la traduction. Des sites mineurs se trouvent une centaine de bases en aval des sites majeurs d'initiation [16]. Deux types d'ARNm correspondant aux deux sites majeurs d'initiation de la transcription ont été décrits [17].

Tableau II

FAMILLE DES GĖNES DE RÉSISTANCE MULTI-DROGUES

\begin{tabular}{|llll|}
\hline & I & II & III \\
\hline Hamster & pgp 1* & pgp2 & pgp3 \\
Souris & $m d r 3^{*}$ (1a) & mdr $1^{*}$ (1b) & mdr2 \\
Homme & $m d r 1^{*}$ & & $m d 2^{* *}$ \\
\hline
\end{tabular}

* confère le phénotype MDR ; * mdr3 pour Borst [17].

$m / s n^{\circ} 5$ vol. 6 , mai 90
Dans les lignées hautement résistantes, il $y$ a très souvent coamplification des gènes $m d r 1$ et $m d r 2$, et il a même été décrit par Borst $e t$ al. [18] une amplification simultanée d'autres gènes ( $m d r 4$ ou gène de la sorcine, $m d r 5$ et $m d r 6)$, dont le rôle dans la résistance aux cytostatiques n'est pas connu.

\section{Expression du gène mar 1}

L'expression du gène $m d r 1$ peut être mesurée soit au niveau de l'ARNm, soit au niveau de la protéine (Tableau III, p. 446). Plusieurs sondes reconnaissant pour la plupart un fragment 5' du gène mdr1 humain sont disponibles. Une hybridation croisée avec le produit du gène $m d r 2$, dont le rôle reste inconnu, peut se produire étant donnée la grande homologie de séquence entre les gènes $m d r 1$ et $m d r 2$. Les anticorps monoclonaux utilisés reconnaissent soit un épitope externe (MRK16), soit un hexapeptide intracytoplasmique présent aux deux extrémités de la molécule (C219). Là encore, leur spécificité peut être prise en défaut, puisque, par exemple, le C219 reconnaît aussi le produit du gène $m d r 2$. Le niveau d'expression du gène $m d r 1$ est le plus souvent estimé par slot blot* ${ }^{*}$ Par rapport à la lignée parentale sensible, les lignées MDR utilisées comme contrôles positifs ont un niveau de résistance très variable, leur DL50 (dose pour laquelle l'inhibition de croissance est de $50 \%$ ) visà-vis de l'adriamycine variant de 3 à 200 fois. Ces différences peuvent rendre compte de l'hétérogénéité des résultats publiés concernant l'expression du gène $m d r 1$ dans des tumeurs de même type.

Plusieurs tissus normaux expriment fortement le gène $m d r 1$ (Tableau $I V$, p. 447) : dans un ordre décroissant, la médullaire des surrénales, le rein, le tube digestif, le foie, le poumon sont riches en P-gp $[19,20]$. L'immunocytochimie montre que cette glycoprotéine est localisée à la surface luminale de ces organes émonctoires : bordure en brosse des

\footnotetext{
* Slot blot: technique d'hybridation moléculaire sur filtre: des échantillons d'ARN sont "buvardés" (blotted) sur un filtre à l'aide d'un appareil délimitant des fentes (slots). Le filtre est ensuite hybridé avec la sonde.
} 
Tableau III

\section{MÉTHODES D'ÉTUDE DE L'EXPRESSION DU GĖNE MDR}

Au niveau de I'ARN messager :

Northern blot

Slot ou Dot blot

Protection contre la nucléase S1

Hybridation in situ

Transcriptase inverse puis amplification enzymatique in vitro (PCR)

\section{RÉFÉRENCES}

13. Azzaria M, Schurr E, Gros P. Discrete mutation introduced in the predictive nucleotide binding sites of the $m d r 1$ gene abolish its ability to confer multidrug resistance. Mol Cell Biol 1989 ; 9 : 5289-97.

14. Willingham MC, Cornwell MM, Cardarelli CO, Gottesman MM, Pastan I. Single cell analysis of daunomycin uptake and efflux in multidrug-resistant and sensitive KB cells : effect of verapamil and other drugs. Cancer Res 1986 ; 46 : 5941-6.

15. Fojo $A$, Lebo $R$, Shimizu $N$, et al. Localization of multidrug resistanceassociated DNA sequences to human chromosome 7. Somatic Cell Mol Genet 1986 ; 12 : 415-20.

16. Chen CJ, Clark D, Ueda K, et al. Genomic organization of the human multidrug resistance (mdr1) gene and origin of P-glycoproteins. J Biol Chem $1990 ; 265$ : 506-14.

17. Rothenberg ML, Mickley LA, Cole $\mathrm{DE}$, et al. Expression of the $m d r 1 / \mathrm{P}-170$ gene in patients with acute lymphoblastic leukemia. Blood 1989 ; $74: 1388-95$.

18. Van der Bliek AM, Baas F, Van der Velde-Koerts, et al. Genes amplified and overexpressed in human multidrug-resistant cell lines. Cancer Res $1988 ; 48: 5927-32$.

19. Fojo A, Ueda K, Salmon DJ, Poplack DG, Gottesman MM, Pastan I. Expression of a multidrug-resistant gene in human tumor and tissues. Proc Natl Acad Sci USA 1987 ; 84 : 265-9.

20. Thiebaut $F$, Tsuruo $T$, Hamada $H$ Gottesman MM, Pastan I, Willingham MC. Cellular localization of the multidrugresistance gene product $\mathrm{P}$-glycoprotein in normal human tissues. Proc Natl Acad Sci USA 1987 ; 84 : 7735-8.

21. Cordon-Cardo C, O'Brien JP, Casals $\mathrm{D}$, et al. Multidrug-resistance gene ( $\mathrm{P}$ glycoprotein) is expressed by endothelial cells at blood barrier sites. Proc Natl Acad Sci USA 1989 ; 816 : 695-8.

22. Goldie JH, Coldman AJ. The genetic origin of drug resistance : implications for Systemic Therapy. Cancer Res 1984; 44 : 3643-53.
SONDES UTILISÉES :

\begin{tabular}{|llll|}
\hline Laboratoire & Origine & Partie reconnue & Taille \\
\hline Ueda & $\begin{array}{l}\text { lignée KB-C2.5 } \\
\text { (humaine) }\end{array}$ & $\begin{array}{l}\text { côté } 5^{\prime} \\
\text { milieu }\end{array}$ & $\begin{array}{l}1 \mathrm{~kb} \\
\text { Roninson }\end{array}$ \\
$\begin{array}{l}\text { lignée KB-C2.5 } \\
\text { Gros }\end{array}$ & $\begin{array}{l}\text { côté } 5^{\prime} \\
\text { foie fœumain) }\end{array}$ & côté $5^{\prime}$ & $1,3 \mathrm{~kb}$ \\
Borst & $\begin{array}{l}\text { lignée HepG2 } \\
\text { Cowan }\end{array}$ & côté $3^{\prime}$ & $0,6 \mathrm{~kb}$ \\
& lignée MCF-7 & côté $3^{\prime}$ & $0,6 \mathrm{~kb}$ \\
\end{tabular}

\footnotetext{
Au niveau de la protéine:

Western blot

Immunocytochimie

Cytoflucrométrie
}

ANTICORPS :

\begin{tabular}{|lll|}
\hline Nom & Épitope reconnu & Laboratoire \\
\hline C219 & $\begin{array}{l}\text { Hexapeptide } \\
\text { intracytoplasmique } \\
\text { (Lignée hamster) }\end{array}$ & $\begin{array}{l}\text { Ling (commercialisé } \\
\text { par Centocor) }\end{array}$ \\
MRK 16 & $\begin{array}{l}\text { 1re boucle externe } \\
\text { (K 562/ADR) }\end{array}$ & Tsuruo (non commercialisé) \\
JSB 1 & $\begin{array}{l}\text { épitope } \\
\text { intracytoplasmique } \\
\text { épitope de surface }\end{array}$ & commercialisé par Tébu \\
Hyb 612 & Hybritech (en développement)
\end{tabular}

des cellules des tubules proximaux rénaux, canalicules biliaires et pancréatiques. Cette technique, en révélant de la P-gp au niveau des capillaires cérébraux et testiculaires [21], laisse penser que l'expression des gènes MDR pourrait participer à l'effet barrière de ces organes.

Le rôle important (détoxifiant ?) de cette protéine membranaire est suspecté devant sa localisation et la conservation de sa structure au cours de l'évolution. Il est probable que la présence de P-gp dans les cellules de mammifères assure un rôle de protection des organismes supérieurs contre des toxines végétales et micro- biennes, et que, dans les cellules cancéreuses, elle assure ce même type de fonction vis-à-vis de certains cytostatiques.

Dans les tissus tumoraux, une expression élevée de P-gp pourrait contribuer à la chimiorésistance initiale observée dans certains cancers (reins, côlon) et pourrait expliquer l'apparition de résistance croisée lors des rechutes. L'échec relatif des chimiothérapies " alternes ", fondées sur le modèle mathématique de mutations somatiques spontanées et aléatoires de Goldie et Coldman [22], serait dû en partie à la résistance croisée de cellules exprimant la P-gp. 
Tableau IV

EXPRESSION DU GĖNE MDR1

\begin{tabular}{|c|c|c|c|}
\hline & Tissus normaux & Tissus tumoraux & \\
\hline$V_{\text {absence }}$ & $\begin{array}{l}\text { Glande surrénale } \\
\text { Côlon } \\
\text { Rein } \\
\text { Foie } \\
\text { Poumon } \\
\text { Ovaire, estomac } \\
\text { moelle osseuse, } \\
\text { rate, ganglions, muscle... }\end{array}$ & $\begin{array}{l}\text { Phéochromocytome } \\
\text { Adénocarcinome colique } \\
\text { Adénocarcinome rénal } \\
\text { Hépatome } \\
\text { Hématosarcome } \\
\left.\begin{array}{l}\text { Neuroblastome } \\
\text { Adénocarcinome ovarien }\end{array}\right\} \\
\text { Mélanome }\end{array}$ & $\begin{array}{l}\text { après } \\
\text { traitement }\end{array}$ \\
\hline
\end{tabular}

Depuis 1985, l'expression du gène mdr1 a été mesurée dans de nombreuses tumeurs, par des méthodes variées, avec des standards (lignées sensitives et résistantes) différents, rendant difficile une analyse globale. Il n'a jamais été retrouvé d'amplification du gène dans ces échantillons tumoraux, même en cas d'expression très augmentée.

On peut regrouper les tumeurs en trois classes (Tableau IV) : celles qui expriment très souvent, même avant traitement, le gène $m d r 1$ : les tumeurs du côlon, du rein, de la glande surrénale, du pancréas, du foie, dérivant de tissus normaux exprimant un taux élevé de P-gp. Celles qui expriment occasionnellement avant traitement, et de façon plus fréquente après chimiothérapie, ce même gène : le neuroblastome et le cancer de l'ovaire [23], les hématosarcomes [24]. Enfin, des tumeurs tels les mélanomes qui semblent ne pas exprimer le gène $m d r 1$, quels que soient le stade évolutif et la chimiorésistance. La situation reste confuse pour plusieurs des cancers les plus fréquents : bronches, sein. Les publications sont en effet contradictoires ou portent sur de trop petits échantillons pour être rapportées.

\section{Régulation du gène mdr1}

In vitro, il est possible d'induire ou d'augmenter l'expression du gène $m d r 1$ dans plusieurs lignées de tumeurs coliques par des inducteurs de différenciation comme le butyrate de sodium [25]. Au cours de cette différenciation induite, l'on peut sui$\mathrm{m} / \mathrm{s} n^{\circ} 5$ vol. 6 , mai 90 vre par hybridation in situ, l'augmentation de l'expression de la P-gp.

Une élévation considérable de l'expression du gène $m d r 1$ est décrite dans les nodules hépatiques prénéoplasiques et néoplasiques induits chez le rat par des carcinogènes (modèle de Solt Farber) [26]. Cette augmentation s'accompagne d'autres modifications cellulaires, comme une augmentation de l'isoforme $\mathrm{d} d u$ cytochrome $\mathrm{P} 450$ et de la glutathionS-transférase, le tout concourant à rendre les cellules plus résistantes aux agressions toxiques, y compris à celles des carcinogènes eux-mêmes.

Dans les lignées continues et les tumeurs après chimiothérapie, la sélection d'une sous-population de cellules tumorales résistantes est plus probable que l'induction de l'expression du gène $m d r 1$ par les cytostatiques. Les relations chimiothérapie expression du gène $m d r 1$ restent cependant à préciser.

L'importance clinique de l'expression de ce gène au niveau des cellules tumorales n'est pas encore parfaitement démontrée. Le fait que des tumeurs notoirement chimiorésistantes, comme les adéno-carcinomes du tube digestif ou les cancers du rein, expriment (et dérivent de tissus exprimant) la P-gp ne doit pas faire conclure hâtivement à l'unique responsabilité de celle-ci : la chimiorésistance est souvent multifactorielle, et d'autres éléments, en particulier la cinétique cellulaire, sont à prendre en compte. Les meilleurs modèles d'étude sont les hématosarcomes habituellement chimiosensibles en début d'évolution, et où l'appari- tion du phénotype MDR est ensuite fréquente. De rares publications (myélomes [27], leucémies [24]), portant sur peu de patients, permettent d'étayer fragilement la responsabilité du gène $m d r 1$ dans la résistance à la chimiothérapie. Les deux difficultés d'interprétation sont l'absence de valeurs de référence et l'usage d'une polychimiothérapie, comprenant souvent des cytostatiques ne se liant pas à la P-gp.

S'il se confirmait que le phénomène MDR peut être rendu responsable de chimiorésistance en cancérologie humaine, il faudrait alors tenir compte du spectre d'action de la Pgp dans le choix des chimiothérapies : recourir à des cytostatiques indépendants du phénotype MDR en cas de résistance et lors de l'emploi de chimiothérapie "alterne ", soit profiter d'une éventuelle sensibilité collatérale, soit essayer d'inhiber la P-gp. Ceci pourrait être effectué, dans le cadre d'essais thérapeutiques de phase I-II, par l'adjonction de modulateurs.

\section{Modulation}

\section{du phénotype MDR}

Le phénotype MDR peut être modulé par de nombreux produits tels le vérapamil, la trifluopérazine, la quinidine, la ciclosporine A et certaines céphalosporines [27] (Tableau $V$, page suivante). Ces agents partagent plusieurs des caractères communs aux substrats de la P-gp, comme la lipophylie et la stéréochimie polycyclique. Dans la majorité des cas, le mécanisme d'action est une inhibition compétitive de l'expulsion du cytostatique [28].

Le vérapamil et la trifluoropérazine ont été utilisés sans réelle efficacité chez des patients dont les tumeurs étaient cliniquement multirésistantes. Ces échecs peuvent être liés, d'une part, à la toxicité propre de ces modulateurs, qui ne peuvent être employés in vivo qu'à des concentrations sub-optimales, et, d'autre part, au fait que ces tumeurs avaient peutêtre d'autres mécanismes de chimiorésistance, la $\mathrm{P}$-gp n'ayant le plus souvent pas été mesurée chez ces patients.

Des essais sont actuellement en cours avec la ciclosporine A (et bientôt 


\begin{tabular}{|ll|}
\hline \multicolumn{2}{|c|}{ Tableau V } \\
MODULATEURS DE LA & P-gp \\
\hline Vérapamil & $6 \mu \mathrm{M}^{*}$ \\
Trifluopérazine & $3 \mu \mathrm{M}$ \\
Quinidine & $3 \mu \mathrm{M}$ \\
Tamoxifène & $3 \mu \mathrm{M}$ \\
Ciclosporine & $1 \mu \mathrm{M}$ \\
Céfopérazone & $1 \mathrm{mM}$ \\
\hline
\end{tabular}

- dose nécessaire in vitro pour contrôler la chimiorésistance.

\section{RÉFÉRENCES}

23. Bourhis J, Goldstein LJ, Riou G, Pastan I, Gottesman MM, Bénard J. Expression of a human multidrug resistance gene in ovarian carcinomas. Cancer Res 1989 ; 49 : 5062-5.

24. Marie JP, Zittoun R, Sikic BI. Multidrug resistance $(m d r 1)$ gene expression in adult acute leukemias : correlations with treatment outcome and in vitro drug sensitivity (soumis à publication).

25. Mickley LA, Bates SE, Richert ND, et al. Modulation of the expression of a multidrug resistance gene ( $m d r 1 / \mathrm{P}$-glycoproteine) by differentiating agents. $J$ Biol Chem 1989 ; 264: 18031-40.

26. Thorgeirsson SS, Huber BE, Sorrell S, Fojo A, Pastan I, Gottesman MM. Expression of the multidrug resistant gene in hepatocarcinogenesis and regenerating rat liver. Science 1987 ; 236, 1120-2.

27. Dalton WS, Grogan TM, Meltzer TM, et al. Drug-resistance in multiple myeloma and non-Hodgkin's lymphoma : detection of $\mathrm{P}$-glycoprotein and potential circumvention by addition of verapamil to chemotherapy. $J$ Clin Oncol $1989 ; 7$ : 415-24.

28. Gosland MP, Lum BL, Sikic BI. Reversal by cefoperazone of resistance to etoposide, doxorubicin, and vinblastine in multidrug resistant human sarcoma cells. Cancer Res 1989 (sous presse).

29. Foxwell BMJ, Mackie A, Ling V, Ryffel B. Identification of the mdr-related P-glycoprotein as a cyclosporine binding protein. Mol Pharmacol 1989 ; 36 : 543-6.

30. Hayes JD, Wolf CR. Role of glutathione transferase in drug resistance. In : Sies H, Kettener B, eds. Mechanism and Biological Consequence of Glutathione Conjugaison. London: Academic press, 1988 : 315-55.

31. Beck WT. Unknotting the complexities of multidrug resistance : the involvement of DNA topoisomerases in drug action and resistance. I Natl Cancer Inst 1989; 81 : 1683-5. avec des molécules proches non néphrotoxiques), utilisable in vivo à des concentrations optimales. Les effets secondaires de ces traitements doivent être suivis attentivement, car nul ne sait ce qu'il en coûte d'inhiber la P-gp présente au niveau de nombreux tissus normaux.

\section{Autres mécanismes biochimiques de résistance à la chimiothérapie}

Dans les modèles in vitro, il est fréquent d'observer l'apparition simultanée de plusieurs mécanismes de résistance, ce qui rend difficile l'évaluation de l'importance respective de chacun d'eux. Il est probable qu'il en soit de même in vivo. Nous ne citerons que deux domaines faisant l'objet d'investigations nombreuses : (a) le métabolisme du glutathion, dont l'enzyme clé est la glutathionS-transférase (GST), qui pourrait jouer un rôle dans la résistance aux agents alkylants et aux anthracyclines en augmentant les mécanismes antioxydatifs de la cellule [30], et (b) les modifications des topo-isomérases, cibles de l'amsacrine, des podophyllotoxines et en partie des anthracyclines [31].

\section{Conclusions}

La découverte de cette famille de glycoprotéines présentes dans certains tissus normaux et tumoraux est certainement importante, en raison de sa conservation de structure au cours de l'évolution des espèces. Son rôle dans les tissus normaux doit être précisé, et sa responsabilité dans les résistances tumorales aux chimiothérapies est en cours d'évaluation clinique. Le caractère très souvent multifactoriel des résistances doit inciter à la prudence, mais il faut souligner que, pour la première fois, le clinicien peut avoir une approche rationnelle de l'obstacle que représente la résistance multiple aux anticancéreux

\section{TIRÉS A PART}

J.-P. Marie.

\section{Summary}

The multidrug resistance phenotype: $M D R$ genes and $P-g P$

The resistance to cytotoxic drugs poses a major obstacle to the ultimate success of cancer therapy. The intrinsic resistance of tumoral cells is one of the major cause of treatment failure. The overexpression of a membrane associated glycoprotein, P - gP, in tumoral cell lines resistant to a wide range of drugs, permitted the description of a "multidrug resistance " (MDR) phenotype. This $\mathrm{P}-\mathrm{gp}$, wich appears to play a role in drug efflux, is encoded by the $m d r-1$ gene in human. The frequent $m d r-1$ gene overexpression in clinically resistant tumors suggests that this gene may be responsible for treatment failure in human cancers. Phase I-II trials, testing $\mathrm{P}-\mathrm{gp}$ modulators, are now in progress. 